\title{
Comparative study of tramadol and diclofenac as analgesic for postoperative pain
}

\author{
Shukla $\mathbf{A K}^{1}$, Srivastav $\mathrm{AK}^{2}$ \\ ${ }^{1}$ Dr. Ajay Kumar Shukla, Assistant Professor, ${ }^{2}$ Dr. Arun Kumar Srivastav, Professor; Both are affiliated with Department \\ of Pharmacology, Gandhi Medical College, Bhopal, MP, India
}

Address for Correspondence: Dr. Ajay Kumar Shukla, Assistant Professor, Department of Pharmacology, Gandhi Medical College, Bhopal, drajay1024@gmail.com

\begin{abstract}
Introduction: Postoperative pain is both distressing and detrimental for the patient. Postoperative pain may be a significant reason for delayed discharge from hospital, increased morbidity and reduced patient satisfaction. Methods: This study is a hospital based prospective, randomized, comparative, observational study over a period of one year. For the purpose of study, patients were randomly allocated equally between two analgesic protocols for patients operated for hydrocele and inguinal hernia. Pain assessment was done by using Visual Analog Scale (VAS). Results: In the first 24,48 and 72 hours of postoperative period, mean VAS scores differed significantly between diclofenac Vs. tramadol $(\mathrm{p}<0.001)$. In patients operated for hernia, in the first 24,48 and 72 hours of postoperative period, mean VAS scores differed significantly between diclofenac Vs. tramadol $(\mathrm{p}<0.001$. In patients operated for hydrocele, in the first 24 hours of postoperative period, mean VAS scores differed significantly $(\mathrm{p}<0.001)$ but in the first 48 and 72 hours of postoperative period, mean VAS scores did not differ significantly between diclofenac Vs. tramadol. Conclusions: Diclofenac provides effective and better analgesia in acute post operative pain than tramadol. Also, tramadol requires more frequent administration than diclofenac.
\end{abstract}

Keywords: Pain assessment, Visual analog scale, Analgesia, Analgesic efficacy, VAS

\section{Introduction}

Postoperative pain may be a significant reason for delayed discharge from hospital, increased morbidity and reduced patient satisfaction [1]. Factors affecting the degree of post-operative pain include the patient's previous experiences and mental preparation (which can be influenced by the surgeon and anaesthetist), intraoperative pain management, the nature and duration of surgery, the site and size of the incision and the extent of surgical trauma [2].

The management of postoperative pain involves assessment of the pain in terms of intensity at rest and activity associated pain, treatment by pharmacological and non pharmacological means as well as monitoring induced side-effects. Besides being physically and emotionally disabling, pain is associated with various physiological effects involving increased perioperative

Manuscript received: $7^{\text {th }}$ Nov 2015

Reviewed: $16^{\text {th }}$ Nov 2015

Author Corrected: $30^{\text {th }}$ Nov 2015

Accepted for Publication: $19^{\text {th }}$ Dec 2015 stress response. The pain causes the patient to remain immobile, thus becoming vulnerable to deep vein thrombosis (DVT), pulmonary atelectasis, muscle wasting and urinary retention. The aims of postoperative analgesia are to achieve pain relief without nausea and drowsiness and to avoid breakthrough pain during convalescence [3].

Repair of inguinal hernia and hydrocele are the two common surgeries performed by general surgeons; convalescence time away from work after such surgery constitutes an important loss of income to both individual and nation [4].

Drugs used for pain can be grouped as simple analgesics (e.g. paracetamol), non-steroidal antiinflammatory drugs (NSAIDs), opioids, and adjuvants (eg, antiepileptics, antidepressants). The cause of pain must also be considered when selecting treatment [2]. 
There is increasing interest in the use of non-steroidal anti-inflammatory drugs (NSAIDs) as analgesics for postoperative pain relief [5]. The aim of using such drugs is not only to reduce the requirements for opioids and thereby the frequency of unwanted side-effects, but also to improve the quality of pain relief [6].

Pain that accompanies inflammation and tissue injury probably results from local stimulation of pain fibers and enhanced pain sensitivity (hyperalgesia), in part a consequence of increased excitability of central neurons in the spinal cord. NSAIDs are particularly effective when inflammation has caused sensitization of pain receptors to normally painless mechanical or chemical stimuli [7]. Diclofenac is a non-steroidal antiinflammatory drug (NSAID) used for postoperative pain relief.

Diclofenac has analgesic, antipyretic, and antiinflammatory activities. Its potency against cyclooxygenase-2 (COX-2) is substantially greater than that of indomethacin, naproxen, or several other NSAIDs. Diclofenac is useful for short-term treatment of postoperative pain, acute musculoskeletal pain, and dysmenorrhea [7].

There are many reasons for preference of intramuscular diclofenac for postoperative pain relief. It has got rapid absorption and rapid onset of action. It is more potent analgesic than aspirin. The selectivity of diclofenac for COX-2 resembles that of celecoxib so that the incidence of serious gastrointestinal adverse effects did not differ between celecoxib and diclofenac in the CLASS trial [8].

Tramadol is a synthetic codeine analog that is a weak $\mu$ opioid receptor agonist. Additionally, it also works by inhibiting reuptake of uptake of norepinephrine and serotonin. In the treatment of mild-to-moderate pain, tramadol is as effective as morphine or meperidine. Tramadol has got $100 \%$ bioavailability by intramuscular route. Tramadol has rapid onset of action. There is no ceiling dose for tramadol; therefore, pain management can be individually tailored to patient/pain response. Thus, it provides additional advantage to prefer it for postoperative analgesia. There is no significant risk of addiction with short term use of tramadol for postoperative pain management. Respiratory depression appears to be less than equianalgesic doses of morphine and is reversible by naloxone. The degree of constipation is less than that seen after equivalent doses of codeine [9].
Postoperative pain is an acute condition. Postoperative pain management is plays an important role for prevention of postoperative complications. Most of the studies done on similar subjects have been done in western population and none of them included Indian subjects, as we are ethnically different from our Caucasian counterpart, therefore, by this study, we want to establish an epidemiological data regarding postoperative analgesia and analgesic being used in our hospital .This study deals with comparative study of tramadol and diclofenac as analgesic in first postoperative 72 hours in patients operated for inguinal hernia and hydrocele. The idea beyond this study is to get an evidence based appropriate analgesic for postoperative pain.

\section{Methods}

This was a hospital based prospective, randomized, comparative, observational study over a period of one year. Subjects of this study were 12-70 years aged male in-patients of a tertiary care hospital who were operated for hydrocele and inguinal hernia. Two analgesic protocols were compared for postoperative pain relief after surgery for hydrocele and inguinal hernia. All the patients were of ASA grade I and II. Each subject was informed in detailed and comprehensive manner about pain assessment scale in the pre-operative period. Only patients who understood the scale and were capable of expressing their pain in terms of the scale were chosen for the study. Written informed consent was taken from every patient before including in the trial. For the purpose of study, equal numbers of patients were randomly allocated the two analgesic protocols. Drug protocol followed in each group:

Group A (67 patients) _ $\quad$ Tramadol $\quad 100 \mathrm{mg}$ intramuscular every 6 hours for 72 hours.

Group B (67 patients) - Diclofenac $75 \quad \mathrm{mg}$ intramuscular every 12 hours for 72 hours.

Same drug preparation of a particular pharmaceutical company was used in each drug group to prevent the difference in the drug response due to difference in formulations. Simple random sampling was done for the allocation of group. Patients were subjected to thorough history, clinical examination, biochemical investigations, and detailed preanaesthetic assessment. Pain assessment was done by using Visual Analog Scale (VAS) [10]. 
No analgesia was given to the subjects in the immediate postoperative period. For the initial post operative 72 hours, analgesic drugs were given according to the drug protocol only. No any other analgesic drug or drug which can interfere with pain perception of the patient was given in initial post operative 72 hours.

Patients operated for inguinal hernia and hydrocele were allocated to receive either tramadol or diclofenac as an intramuscular injection after significant pain (VAS > 3). The time interval taken is arbitrary and according to the duration of action of anesthesia used. Then initial postoperative pain assessment was done. Subject was kept under observation till the onset of pain. After the onset of pain, detailed evaluation of the pain was done. Patient was kept under observation for the whole period of initial post operative 72 hours for any post operative complication, which in case, if present, was excluded from the study.

Patients were regularly assessed for the post operative pain relief thrice a day (at 8 hours, 16 hours, 24 hours, 32 hours, 40 hours, 48 hours, 56 hours, 64 hours and 72 hours assigning entry of patient in ward after surgery as 0 hours).Worst pain in between the two readings was asked to assess whether there was increase in pain intensity in between this period. If there was marked increase in the pain intensity in the inter dose interval i.e. increase in the visual analog scale score by more than $10 \mathrm{~mm}$, additional dose of analgesic agent was to be administered and such patients was to be excluded from the study. Appropriate steps were taken to prevent occurrence of any adverse effect, which in case, if present, were reported.

The study was done with the intention to treat. Any side effect was carefully noted. Compliance to the drug was assured since the drug was injected intramuscularly under direct supervision in the hospitalized patient.

The data so collected was tabulated and subjected to statistical analysis using Statistical Package for Social Sciences (SPSS). The confidence limit of the study was kept at $95 \%$, hence a "p" value less than 0.05 indicated a statistically significant association.

\section{Results}

- In the first 24,48 and 72 hours of postoperative period, mean VAS scores differed significantly between diclofenac Vs. tramadol $(\mathrm{p}<0.001)$. (Table 1$)$

- In patients operated for hernia, in the first 24,48 and 72 hours of postoperative period, mean VAS scores differed significantly between diclofenac Vs. tramadol ( $\mathrm{p}<0.001)$. (Table 2)

- In patients operated for hydrocele, in the first 24 hours of postoperative period, mean VAS scores differed significantly $(\mathrm{p}<0.001)$ but in the first 48 and 72 hours of postoperative period, mean VAS scores did not differ significantly between diclofenac Vs. tramadol ( $p=0.75 \& \mathrm{p}=0.76)$. (Table 3 )

Table No 1: Mean VAS scores at 24, 48 and 72 hours in two drug groups operated for inguinal hernia as well as hydrocele

\begin{tabular}{|c|c|c|c|}
\hline Duration of Treatment & Value & Tramadol & \\
\hline \multirow{3}{*}{24 hours } & Mean & $2.5 \pm 0.24$ & $2.01 \pm 0.10$ \\
\hline & T-value & \multicolumn{2}{|l|}{14.23} \\
\hline & $\mathrm{P}$ - value & \multicolumn{2}{|l|}{$<0.001$} \\
\hline \multirow{3}{*}{48 hours } & Mean & $1.8 \pm 0.33$ & $1.59 \pm 0.16$ \\
\hline & T-value & \multicolumn{2}{|l|}{4.62} \\
\hline & $\mathrm{P}$ - value & \multicolumn{2}{|l|}{$<0.001$} \\
\hline \multirow{3}{*}{72 hours } & Mean & $1.04 \pm 0.32$ & $0.87 \pm 0.19$ \\
\hline & T-value & \multicolumn{2}{|l|}{3.63} \\
\hline & $\mathrm{P}$ - value & \multicolumn{2}{|l|}{$<0.001$} \\
\hline
\end{tabular}


Table No 2: Mean VAS scores at 24, 48 and 72 hours in two drug groups operated for inguinal hernia

\begin{tabular}{|c|c|c|c|}
\hline Duration of Treatment & Value & Tramado & Diclofenac \\
\hline \multirow{3}{*}{24 hours } & Mean & $2.5 \pm 0.04$ & $2.01 \pm 0.20$ \\
\hline & T-value & \multicolumn{2}{|l|}{11.72} \\
\hline & P-value & \multicolumn{2}{|l|}{$<0.0001$} \\
\hline \multirow{3}{*}{48 hours } & Mean & $2.0 \pm 0.27$ & $1.59 \pm 0.49$ \\
\hline & T-value & \multicolumn{2}{|l|}{7.88} \\
\hline & P-value & \multicolumn{2}{|l|}{$<0.0001$} \\
\hline \multirow{3}{*}{72 hours } & Mean & $1.12 \pm 0.2$ & $1.01 \pm 0.13$ \\
\hline & T-value & \multicolumn{2}{|l|}{5.02} \\
\hline & P-value & \multicolumn{2}{|l|}{$<0.0001$} \\
\hline
\end{tabular}

Table No 3: Mean VAS scores at 24, 48and 72 hours in two drug groups operated for hydrocele.

\begin{tabular}{|c|c|c|c|}
\hline Duration of Treatment & Value & Tramadol & enac \\
\hline \multirow{3}{*}{24 hours } & Mean & $2.45 \pm 0.25$ & $2.01 \pm 0.11$ \\
\hline & T-value & \multicolumn{2}{|l|}{8.32} \\
\hline & $\mathrm{P}$ - value & \multicolumn{2}{|l|}{$<0.0001$} \\
\hline \multirow{3}{*}{48 hours } & Mean & $1.59 \pm 0.14$ & $1.51 \pm 0.16$ \\
\hline & T-value & \multicolumn{2}{|l|}{1.81} \\
\hline & $\mathrm{P}$ - value & \multicolumn{2}{|l|}{0.075} \\
\hline \multirow{3}{*}{72 hours } & Mean & $0.81 \pm 0.33$ & $0.68 \pm 0.08$ \\
\hline & T-value & \multicolumn{2}{|l|}{2.04} \\
\hline & $\mathrm{P}$ - value & \multicolumn{2}{|l|}{0.076} \\
\hline
\end{tabular}

\section{Discussion}

Diclofenac, ketorolac \& tramadol are equianalgesic in postoperative pain relief and duration after minor surgical procedures [11]. Analgesic efficacy of oral tramadol \& oral diclofenac for post tonsillectomy pain relief was found to be same in an RCT [12]. In contrast to this our observation concludes that diclofenac and tramadol, both have analgesic effect but analgesic efficacy of diclofenac in first 24 hours is significantly greater than tramadol for postoperative pain.

Diclofenac is a NSAID, widely used to relieve pain with or without inflammation. NSAIDs inhibit the enzyme cyclooxygenase and thereby, inhibit the synthesis of prostaglandins which are the major mediators responsible for pain and inflammation. NSAIDs also inhibit leukocytes migration \& function and reduce the formation of superoxide radicals [13]. Thus, NSAIDs become particularly useful in conditions where a degree of tissue inflammation contribute to pain. Postoperative nociceptive impulses are generated not only by the surgical procedure itself, but also by the action of proteolytic and inflammatory agents released intro wound tissues [14]. Thus, the mechanism of action of NSAIDs justify our observation of a greater analgesic effect than that of tramadol .

Tramadol has analgesic efficacy due to weak $\mu$ agonistic action with additional mechanism of action i.e. the inhibition of reuptake of both 5-HT and noradrenaline together with presynaptic stimulation of 5-HT release [15].Tramadol does not have any effect on action of prostaglandins.

Further, it has been shown that tramadol is effective in mild to moderate pain than in severe post operative pain [16]. This correlates with the fact that there is no significant difference in the analgesic efficacy of tramadol from diclofenac after 48 and 72 hours in patients operated for hydrocele, since, surgery for hydrocele produces minimal tissue injury and pain after 
24 hours is of mild to moderate intensity. First 24 hours of postoperative period is associated with severe pain and significant difference in the analgesic efficacy of tramadol from diclofenac was found.

For moderate to severe pain, oral tramadol $100 \mathrm{mg}$ has NNT of 4.8 in comparison to oral diclofenac $75 \mathrm{mg}$ and $100 \mathrm{mg}$ of 2.7 and 1.8 respectively [17]. NNT is numbers needed to treat with an additional analgesic due to pain relief less than $50 \%$. Thus, a high NNT of tramadol reflects lesser efficacy than diclofenac. It supports our observation of greater efficacy of diclofenac over tramadol in first 24 hours in hydrocele surgery and in 48 and 72 hours also after surgery for hernia.

Central response to stimuli may be modulated by NSAIDs-induced inhibition of prostaglandin synthesis in the spinal cord [18].Single doses of tramadol and hydrocodone-acetaminophen in 68 patients with softtissue pain found significantly lower pain scores in patients receiving hydrocodone-acetaminophen, even using an inadequate dose of $5 \mathrm{mg}$ of hydrocodone with $500 \mathrm{mg}$ of acetaminophen [19]. Tramadol also has proved ineffective for postoperative orthopedic pain [20].

Further, the need of four injections of tramadol in contrast to twice injections of diclofenac in a day increases the patient's inconvenience.

Thus, we conclude that diclofenac provide effective and better analgesia in immediate post operative pain than tramadol. Also, tramadol needs more frequent administration. Further studies can be planed to establish the time of shifting from injectable to oral route and total duration of analgesic requirement.

\section{Conclusion}

Diclofenac provides effective and better analgesia in acute post operative pain than tramadol. Also, tramadol requires more frequent administration than diclofenac.

\section{Funding:Nil. Conflict of interest: Nil. Permission for IRB: Yes}

\section{Abbreviations}

- NNT-numbers needed to treat

- tNSAID-traditional non steroidal anti-inflammatory drug
- NSAID- non steroidal anti-inflammatory drug

- mm- millimetre

- VAS-Visual Analog Scale

- 5-HT-5-Hydroxytryptamine

- RCT-Randomized controlled trial

- h-hours

- ASA- American Society of Anesthesiologists

\section{References}

1. Ryhänen P, Adamski J, Puhakka K, Leppäluoto J, Vuolteenaho O, Ryhänen J. Postoperative pain relief in children. A comparison between caudal bupivacaine and intramuscular diclofenac sodium. Anaesthesia. 1994 Jan;49(1):57-61.

2. Rahman, M.H. and Beattie, J. Managing postoperative pain. Pharmaceutical Journal. July 2005; Vol. $275 ; 145-8$.

3. Jarrett PEM. Day case surgery. Surgery 1995; 13(1): $5-7$.

4. Wantz GE. Ambulatory hernia surgery. Br J Surg. 1989 Dec;76(12):1228-9.

5. Munro HM, Riegger LQ, Reynolds PI, Wilton $\mathrm{NC}$, Lewis IH. Comparison of the analgesic and emetic properties

of ketorolac and morphine for paediatric outpatientstra bismus surgery. Br J Anaesth. 1994 Jun;72(6):624-8.

6. Moffat AC, Kenny GN, Prentice JW. Postoperative nefopam and diclofenac. Evaluation of their morphinesparing effect after upper abdominal surgery.

Anaesthesia. 1990 Apr;45(4):302-5.

7. Goodman \& Gilman's The Pharmacologic Basis of Therapeutics - 11th Ed.New York: McGraw-Hill; 2006.

8. Jüni P, Rutjes AW, Dieppe PA. Are selective COX 2 inhibitors superior to traditional non steroidal antiinflammatory drugs? BMJ. 2002 Jun 1;324(7349):12878.

9. Duthie DJ. Remifentanil and tramadol. Br J Anaesth. 1998 Jul;81(1):51-7.

10. Gallagher EJ, Bijur PE, Latimer C, Silver W. Reliability and validity of a visual analog scale for acute abdominal pain in the ED. Am J Emerg Med. 2002 Jul;20(4):287-90. 
11. Swadia VN, Shah MB. Comparative evaluation of ketorolac, tramadol and diclofenac for postoperative pain relief in minor surgical procedures Indian Journal of Anaesthesia. 1999 Oct; 43(5): 64-6

12. Miller C, Newton SE. Pain perception and expression: the influence of gender, personal selfefficacy, and lifespan socialization. Pain Manag Nurs. 2006 Dec;7(4):148-52.

13. Rational use of NSAIDs for musculoskeletal disorders. Drug Ther Bull. 1994 Dec 15;32(12):91-5.

14. Tverskoy M, Cozacov C, Ayache M, Bradley EL Jr, Kissin I. Postoperative pain after inguinal herniorrhaphy with different types of anesthesia. Anesth Analg. 1990 Jan;70(1):29-35.

15. Vickers MD, Paravicini D. Comparison of tramadol with morphine for post-operative pain following abdominal surgery. Eur J Anaesthesiol. 1995 May;12(3):265-71.

16. Demiraran Y, Kocaman B, Akman RY. A comparison of the postoperative analgesic efficacy of single-dose epidural tramadol versus morphine in children. Br J Anaesth. 2005 Oct;95(4):510-3. Epub 2005 Aug 12.

17. Oxford League Table of Analgesic Efficacy [Internet].

http://www.medicine.ox.ac.uk/bandolier/booth/painpag/ Acutrev/Analgesics/lftab.

18. White PF. The role of non-opioid analgesic techniques in the management of pain after ambulatory surgery. Anesth Analg. 2002 Mar;94(3):577-85.

19. Turturro MA, Paris PM, Larkin GL. Tramadol versus hydrocodone-acetaminophen in acute musculoskeletal pain: a randomized, double-blind clinical trial. Ann Emerg Med. 1998 Aug;32(2):139-43.

20. Stubhaug A, Grimstad J, Breivik H. Lack of analgesic effect of 50 and $100 \mathrm{mg}$ oral tramadol after orthopaedic surgery: a randomized, doubleblind,placebo and standard active drug comparison. Pain. 1995 Jul;62(1):111-8.

\section{How to cite this article?}

Shukla AK, Srivastav AK. Comparative study of tramadol and diclofenac as analgesic for postoperative pain. Int J Med Res Rev 2015;3(11):1311-1316. doi: 10.17511/ijmrr.2015.i11.238. 